\title{
COLLIDER PHYSICS
}

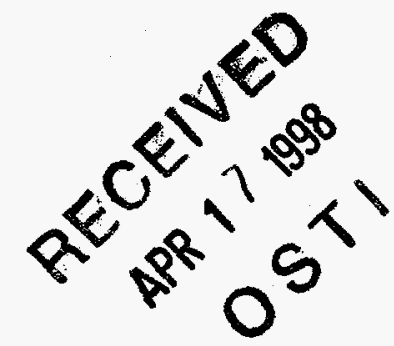

DOEER/40621-TT/

\section{DISCLAIMER}

This report was prepared as an account of work sponsored by an agency of the United States Government. Neither the United States Government nor any agency thereof, nor any of their employees, makes any warranty, express or implied, or assumes any legal liability or responsibility for the accuracy, completeness, or usefulness of any information, apparatus, product, or process disclosed, or represents that its use would not infringe privately owned rights. Reference herein to any specific commercial product, process, or service by trade name, trademark, manufacturer, or otherwise does not necessarily constitute or imply its endorsement, recommendation, or favoring by the United States Government or any agency thereof. The views and opinions of authors expressed herein do not necessarily state or reflect those of the United States Government or any agency thereof.

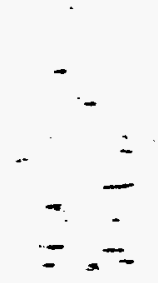

$-\overline{-}$ 


\section{DISCLAIMER}

Portions of this document may be illegible electronic image products. Images are produced from the best available original document. 


\section{Contents}

1 Introduction $\quad 3$

2 Personnel 3

3 The $D \varnothing$ Experiment at Fermilab 6

3.1 Physics......................... 7

3.1.1 Supersymmetry: Wino-Zino to tri-leptons ..... 7

3.1 .2 Leptoquarks................... 8

3.1 .3 Other Physics ................... 17

3.2 Hardware and Software Responsibilities . . . . . . . . 18

3.2 .1 Wino/Zino Triggers for Run $1 \mathrm{~B} \ldots \ldots \ldots$

3.2.2 Level-1 and Level-2 Trigger ........... 19

3.2.3 Level-2 Software - Recent Student Contributions . . . 21

3.2.4 Study of Electron Clustering Algorithms ....... 24

3.2.5 Global Monitoring Shift ............ 25

3.2 .6 Data Processing . . . . . . . . . . . . 29

$3.2 .7 \mathrm{D} \emptyset \mathrm{Upgrade} \ldots \ldots \ldots \ldots . \ldots . \ldots . \ldots 29$

3:2.8 DØ Data Acquisition System Efforts ........ 30

4. The SD̄C Experiment at SSC 35

5 Future:-

$\dot{6}$ Sümmary · 41 


\section{Introduction}

For the past several years our group was in the fortunate position to enjoy both the exciting turn-on and maiden run (run 1A) of the $D \emptyset$ experiment at Fermilab, while also contributing to the design and detector development of the SDC experiment at the SSC. Unfortunately, the SSC was canceled, but we feel our efforts were not wasted - in fact were quite successful and significant. Further, with $D \emptyset$ currently collecting data at an unprecedented rate at $\sqrt{s}=1.8 \mathrm{TeV}$ (run $1 \mathrm{~B}$ ), and with a data set around one inverse femtobarn of data expected by the end of the century (run 2), the future looks great!

Since this is the third of a three year funding period, our report will contain a summary of the highlights for the past three years in addition to a more detailed account of the past year's activities. Sections will include work related to $D \emptyset$ (our main activity), plus a summary of results from our SSC project. Also included, is a section that introduces the members of our group for the past three years and outlines their activities. These activities are then described in more detail in the body of the report.

\section{Pérsonnel}

The members of our group currently include Professor Russ Huson, Assistant Professor James White, Post Doctoral Research Associate Doug Norman and graduate students Djoko Wirjawan, Taylor Goss and David Brookes. Students Wirjawan and Goss, and Post-Doc Doug Norman are all in residence 
at Fermilab. A brief summary of the activities of both our current and past (past three years) members is now given.

Student Djoko Wirjawan arrived at Fermilab in September 1993 and has already mastered the $D \emptyset$ software system. He has already contributed in Level-2 trigger development and is playing a major role in developing an H-Matrix technique to identify tau leptons. His thesis will be to search for Wino-Zino to tri-leptons for run $1 \mathrm{~B}$.

Student Taylor Goss arrived in January 1994. Before that, he worked on our SSC detector program while finishing classes. Since arriving at Fermilab, Taylor has already contributed to Level-2 software development, and has quickly become comfortable in the $D \emptyset$ software system. His thesis will be to search for Wino-Wino, and Wino-Zino to di-leptons.

Student David Brookes is currently finishing class work. He's made significant contributions to our SSC detector project and future activities will be determined after passing the qualifier exam.

Research Associate Doug Norman became a member of our group last spring. His thesis - "A Search for First Generation Scalar Leptoquarks at $\sqrt{s}=1.8 \mathrm{TeV}$ with the $\mathrm{D} \emptyset$ Detector" - lead to the first $\mathrm{D} \emptyset$ physics paper. He will be stationed at Fermilab beyond run 1B to begin preparation for run 2 scheduled to begin in 1998. In addition to guiding our students, he is currently finishing analysis of a search for second generation leptoquarks, optimizing tow $E_{t}$ electron $\mathrm{ID}$, optimizing low $E_{t}$ lepton triggers (needed for wino-zino and wino-wino physics) and developing software for electron ID using the transition radiation detector for run $1 B$. After run $1 B$, he will contribute heavily to the detector upgrade. 
This past year, two members of our group moved up the career ladder. Research Associate Amber Boehnlein joined the Fermilab staff last March. While a member of our group (starting June 1991), Amber was a central participant in the $D \emptyset$ trigger development, tuning, maintenance and monitoring. She worked closely with Jim Linnemann's (Michigan State) group to develop the highly successful level 2 trigger system that was instrumental to the success of $D \emptyset$ 's maiden run. She is also a key member of the new phenomena group, representing its triggering needs on the trigger board.

Research Associate Mei Gui was funded through the Texas National Research Laboratory Commission (TNRLC) to work on detector development for the SSC. Following the demise of the SSC, Mei accepted a position at Prairie View A\&M University. Her work resulted in a very successful program to develop a liquid scintillator based, fiber calorimeter for use at the SSC. Before leaving our group, Mei also contributed to the $D \emptyset$ data processing effort.

Research Associate Jay Wightman was a member of our group when we first joined $D \emptyset$ (spring 1990). He is now a very active member of $D \emptyset$ within the Iowa State group. While with our group, Jay made tremendous contributions in the commissioning, testing and monitoring of the $D \emptyset$ data acquisition system. His work with Marvin Johnson's (of Fermilab) group was critically important to the success of DØ's maiden run. While a member of our group $\bar{p}$, hē also began a very interesting analysis of high $P_{t}$ events with Henryk Piekarz of Florida State University. Jay was and is also one of the "Global Experts" who are responsible for keeping the data acquisition system running when collecting data. 
Earlier students in our group have also successfully finished their projects. Kedarnath Kotha completed his Master's degree last year and is now employed in the electronics industry. His thesis, titled "Track Detection: an MCM Approach" - 1993, describes the design of a chip with the circuitry needed for a fast triggering scheme conceived for the future fiber tracker in DØ. Greg Dudgeon also completed his Master's degree last summer title "Liquid Scintillator Sampling Calorimetry", 1994. His work describes the successful design, construction and testing (in FNAL test beam) of two lead-liquid scintillating fiber calorimeter prototypes. He is now employed in Chicago in the defense industry.

\section{The D $\emptyset$ Experiment at Fermilab}

The $\mathrm{D} \emptyset$ experiment, whose first data run (1A) was completed last year (April 92 -May 93), has already been a productive success. Having accumulated about $15 \mathrm{pb}^{-1}$ data in the first run, three papers have already been published and a large number of topics are nearing publication. This progress is exceptional given the short time since the end of the run and the fact that many thought the first run would be mainly an engineering run for $D \emptyset$. The experiment is now collecting data from run $1 \mathrm{~B}$, that is scheduled to last through December 1995 . Over $20 b^{-1}$ have already been collected for this run, with an anticipated $150 \mathrm{pb}^{-1}$ possible by the end of 1995 .

Our group has contributed strongly to this effort in all phases of the experiment ineluding hardware and software development, test beam, physics analysis and shift duty. In the following, we summarize the highlights of our 
contributions for the past three years.

\subsection{Physics}

\subsubsection{Supersymmetry: Wino-Zino to tri-leptons}

The Wino-Zino $\rightarrow$ tri-lepton analysis has been one of the main activities of our group the past couple years. Now one of the major new phenomena search analyses in both $D \emptyset$ and CDF, it was initiated by our group based on a paper by Arnowitt and Nath (1987). Briefly, the lightest chargino $\widetilde{W_{1}}$ and second lightest neutralino $\widetilde{Z_{2}}$ can be produced together via a virtual $W$ boson. Although weakly produced, the decay modes can result in a tri-lepton signal with little hadronic activity. This allows a fairly sensitive method for detecting these objects at the Tevatron since there is little standard model background.

Members of our group are working on this topic jointly with members of the U.T. Arlington group. Our first results were presented (by J.T. White) at the 9th Topical Workshop on Proton-Antiproton Collider Physics, Tsukuba, Japan (Oct. 1993).

Details of the analysis have now been published in several conference proceedings and will not be repeated here. However, the selections and results for each channel from our first analysis is summarized in Table 3.1.1. The one ee $\mu$ event passing all selections is possibly a $Z^{0} \gamma$ event with the $Z^{0}$ decaying to a tau pair, which then decay leptonically, while the gamma produces a fake electron. A view of the event is shown in Fig. 1. The main result, a limit on cross section times branching fraction to tri-leptons, is shown in Fig. 2 
This limit was based on approximately $2 / 3$ of the data from run $1 \mathrm{~A}$, and a full analysis is now nearing completion as the $\mathrm{PhD}$ work of U.T. Arlington student Mark Sosebee.

These and updated results have been presented at the following conferences and can be found in the proceedings of some of the conferences indicated by "**":

1) Pbar P Workshop KEK J. White New Phenom. 10/18/93 *

2) L. Sawyer - SUSY'94 - Wino/Zino searches with tri-leptons

3) Moriond-Electroweak Merritt New Phenom. 3/12/94 *

4) La Thuile (Italy) Lueking New Phenom. $3 / 06 / 94$

5) Washington APS Baden TOP and NEW (invited talk) 4/18/94

6) Washington APS M. Sosebee SUSY search 4/18/94

7) S. Blessing - DPF'94 - wino/zino *

8) MSU HEP Wkshp (Moscow) S. Willis - New Phenomena 9/16/94

9) Madison Symp. '94 Boehnlein SUSY talk 4/11/94

10) U.S.-Polish HEP Wkshp A. White - TOP and SUSY $9 / 21 / 94$

\subsubsection{Leptoquarks}

The first_physics publication from $D \emptyset$ was a limit on the existence of first generation leptoquarks. This paper was based on the $\mathrm{PhD}$ work of Doug Norman as a University of Maryland student. Doug is now a member of our group and over the past several months, he has extended his work to include second generation leptoquarks. Since this is a major contribution of our group that has just recently reached the maturity needed for external 


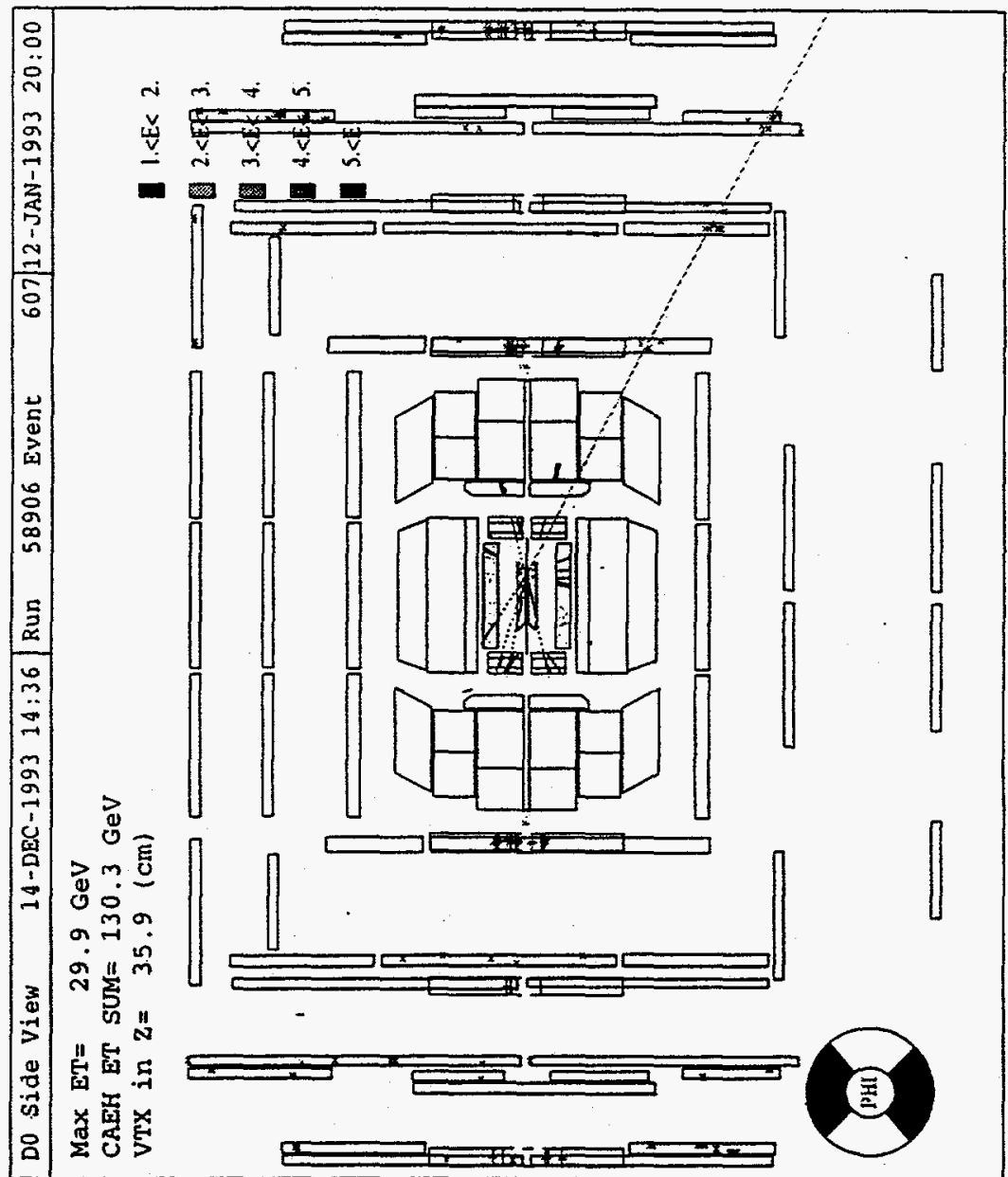

Figure 1:-Trilepton $e e \mu$ candidate from the $\mathrm{D} \emptyset$ experiment. The muon has $\eta=1.1$ and the two electron candidates are at $\eta=2.0$ and 1.9 and can be seen as clusters in the endcap. 


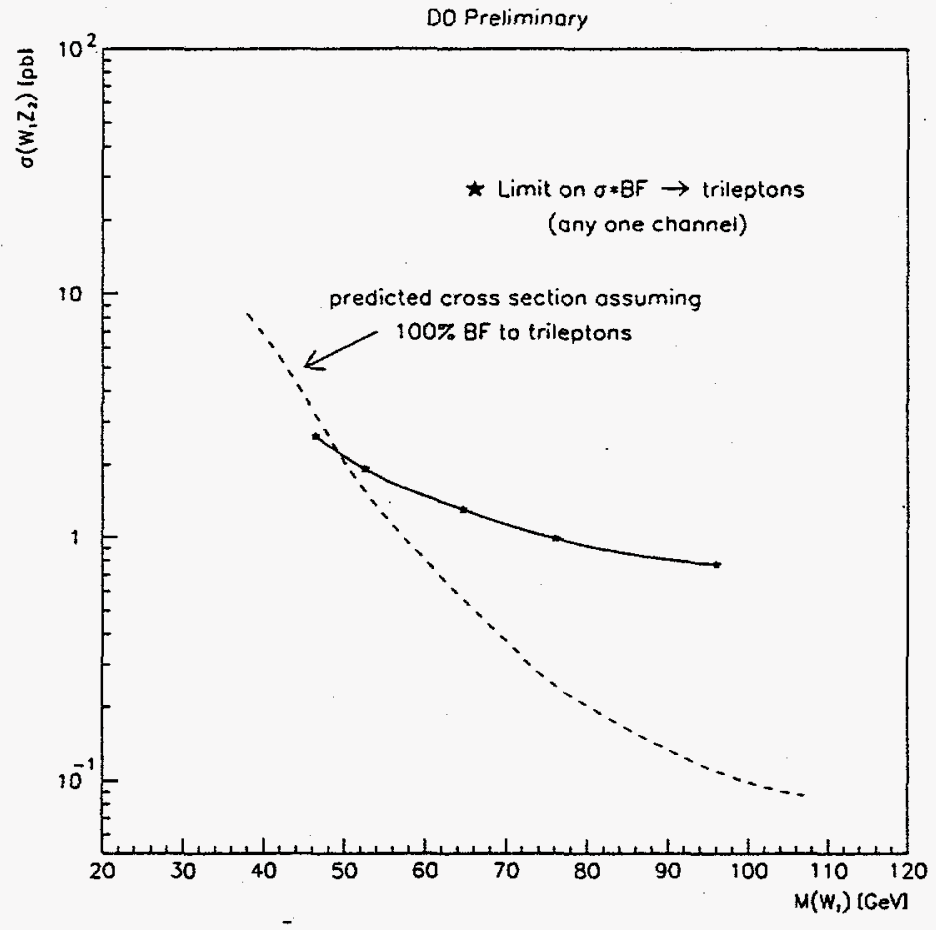

Figure 1: The solid curve represents the $95 \%$ limit on $\sigma \times B F \rightarrow$ tri-leptons for any one channel. The dashed curve represents the theoretical prediction for any one ziode for the case where both the $\widetilde{W}_{1}$ and $\widetilde{Z}_{2}$ decay with $100 \%$ branching fraction to leptons. 
presentation, it will now be discussed in fair detail. These results were also presented by Doug at DPF '94.

The present search for leptoquarks at $D \emptyset$ is for second generation leptoquarks which couple exclusively to muons, muon neutrinos, and $c$ and $s$ quarks. Since leptoquarks are produced primarily in pairs in $\mathrm{p} \overline{\mathrm{P}}$ collisions, the possible signatures for second generation leptoquarks are: 1) two muons plus at least two jets; 2) one muon plus missing transverse energy ( $\mathscr{E}_{T}$ ) from a muon neutrino, plus at least two jets; and 3) $\mathscr{E}_{T}$ plus two or more jets. The $E_{t}$ distributions of the jets, leptons, and, $\mathbb{E}_{T}$ are harder than those of the major backgrounds from $Z^{0}$ and $W$ plus jet production, even for leptoquarks with masses less than the mass of the $\mathrm{Z}^{0}$ and $\mathrm{W}$ bosons because of the transverse boost of the leptoquarks produced in pairs. This is why experimentally leptoquarks are interesting; if they exist at low enough masses they should be easy to detect and are relatively background free.

Backgrounds become less of a problem when there are more leptons in the final state from the decay of pairs of leptoquarks. For the present search, only signatures that have at least one muon were studied. For the signature with two muons, two muons with $\mathrm{P}_{t}$ greater than $25 \mathrm{GeV} / \mathrm{c}$ and at least two jets with $E_{t}$ greater than $25 \mathrm{GeV}$ were required. At least one muon had to be a high quality muon, and at least one muon had to be central (have a pseudorapidity $\eta$ such that $|\eta|<1.0)$. Additional kinematic cuts were applied to reject $Z^{0} \rightrightarrows \mu^{-} \mu^{-}$plus jets events. No events were seen in this channel from $12.3 \mathrm{pb}^{-1}$ of integrated luminosity. The expected background was estimated to be about 0.413 events. For the single muon channel, one quality central muon with $\mathrm{P}_{t}$ greater than $20 \mathrm{GeV} / \mathrm{c}$ was required. Also required were at 
least two jets with $\mathrm{E}_{t}$ greater than $25 \mathrm{GeV}$ and $\mathbb{E}_{T}$ greater than $25 \mathrm{GeV}$. Cuts designed to eliminate $W^{0} \rightarrow \mu \nu$ plus jets background were used leaving no events in the single muon plus jets plus $\mathbb{E}_{T}$ channel. The expected number of background was estimated to be 0.97 events. The event selection in both channels included a trigger cut. The trigger requirements were one muon with $\mathrm{P}_{t}$ greater than $8 \mathrm{GeV} / c$ and a jet with $\mathrm{E}_{t}$ greater than $15 \mathrm{GeV}$ for the software level of the trigger. At the hardware level of the trigger, one muon with $\mathrm{P}_{t}$ greater than $3 \mathrm{GeV} / \mathrm{c}$ and one jet tower $(\eta \times \phi=0.2 \times 0.2)$ with $\mathrm{E}_{t}$ greater than $5 \mathrm{GeV}$ were required.

Observing no events, an upper limit on the cross section as a function of mass for leptoquark pair production can be determined from the upper limits on the number of events expected for leptoquarks of a given mass, the integrated luminosity, and the detection efficiency for leptoquarks with the given mass. The efficiency for detecting second generation leptoquark events was determined from studies of the data and a Monte Carlo simulation. The kinematic acceptance for leptoquarks was derived from leptoquark Monte Carlo. The physics generator used was ISAJET [1]. The generated events were processed with a $D \emptyset$ version of GEANT [2]. The simulated events were then processed with the standard $D \emptyset$ reconstruction code. The muon identification and tracking efficiencies and leptoquark trigger efficiency were defermined from a study of the data and Monte Carlo. The total muon identification and tracking efficiency for the cuts used on the two muons in the two muon event selection is $35.9 \%$. The corresponding efficiency for the single muon in the single muon signature event selection is $30.4 \%$. The trigger efficiency for the two muon leptoquark signal is estimated to be $86 \%$, 
and for the single muon leptoquark signal the efficiency.is $67 \%$. The total detection efficiency for the two muon leptoquark signature is $0.27 \%$ at a mass of $45 \mathrm{GeV} / \mathrm{c}^{2}$ and increased to $7.1 \%$ at a mass of $250 \mathrm{GeV} / \mathrm{c}^{2}$. The detection efficiency for the single muon leptoquark signature is $0.10 \%$ and $4.56 \%$ for respective masses of 45 and $200 \mathrm{GeV} / \mathrm{c}^{2}$. The upper limit on the expected number of events comes from Poisson statistics where the total errors, systematic and statistical, from the analysis are folded into the Poisson PDF function [3]. The total error was about $30 \%$ for the two muon leptoquark signal analysis and $20 \%$ for the single muon leptoquark analysis. The systematic errors were dominated by the uncertainty in the muon momentum resolution. The upper limit on the cross section calculated from the $95 \%$ C.L. upper limit on the number of expected events with no events seen is shown in Fig. 3 for the two muon signature. This limit is actually the limit on the cross section times the branching ratio squared, since the number of expected events in this channel is proportional to the square of the branching ratio, one factor of $\beta$ for each muon. The dashed curve in Fig. 3 is the theoretical prediction of the pair production cross section for scalar leptoquarks times $\beta^{2}$ for $\beta=1$ [4]. The intersection of these two curves gives the lower limit on the mass for pair produced second generation scalar leptoquarks for $\beta=1$ which is $\overline{9} 7-\mathrm{GeV} / \mathrm{c}^{2}$. By moving the theory curve down, according the the value of $\beta$, the mass limit vs $\beta$ can be determined.

No limit from the single muon leptoquark signature could be determined; however, the detection efficiency for this signature can be combined with the detection efficiency from the two muon signature to get a combined limit 


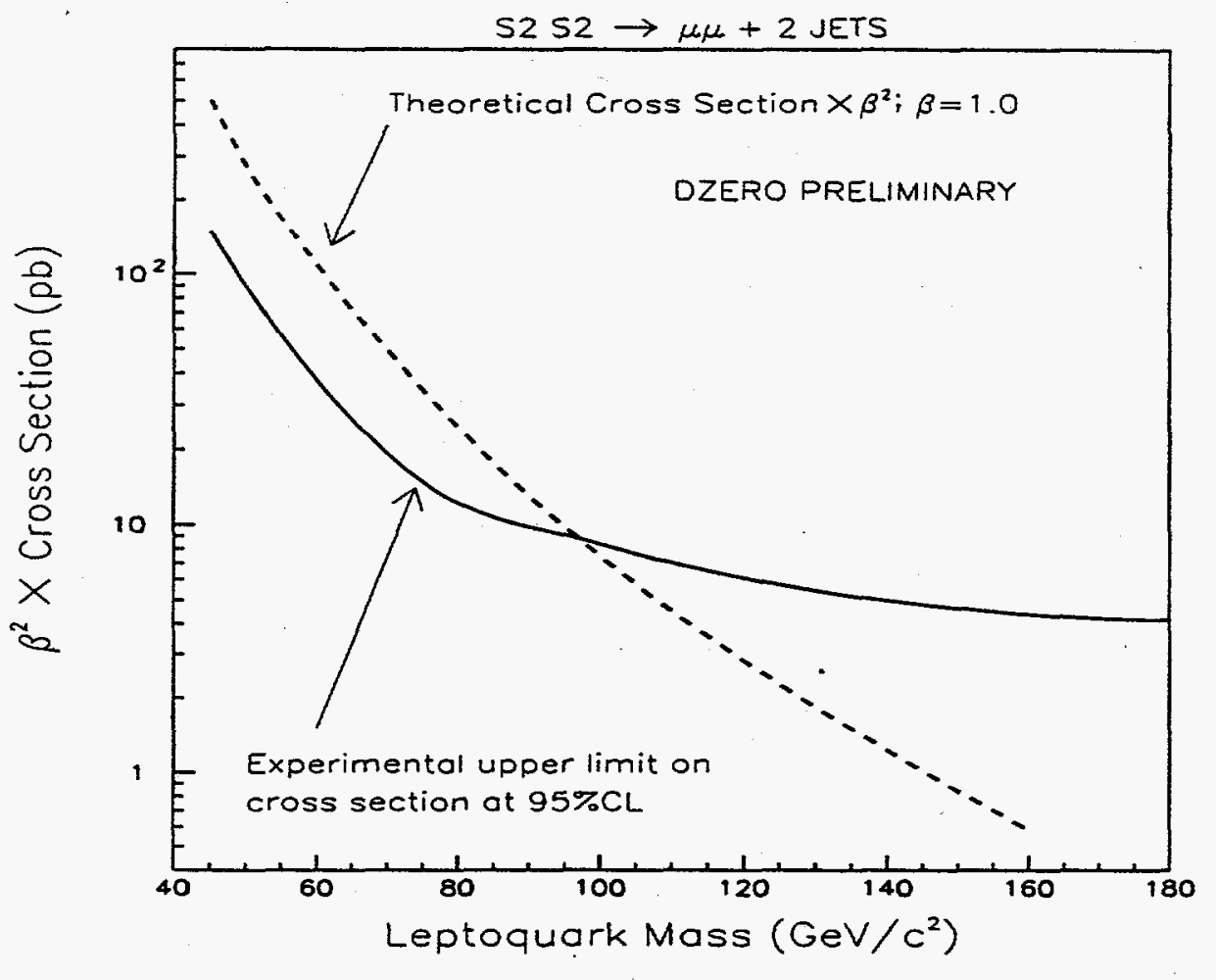

Figure 3: The 95\% CL experimental upper limit times $\beta^{2}$ for the two muon plus two jet signature overlaid with the theoretical cross section times $\beta^{2},(\beta=\overline{1})$ for pair production of scalar leptoquarks 
according to equation 1 .

$$
\sigma_{\text {combined }}(\beta)=\frac{\frac{\beta^{2} \bullet N_{\mu \mu}+2 \beta(1-\beta) \bullet N_{\mu \nu}}{\beta^{2}+2 \beta(1-\beta)}}{12.3 p b^{-1} \cdot\left(\beta^{2} \bullet \epsilon_{\mu \mu}^{L Q}+2 \beta(1-\beta) \cdot \epsilon_{\mu \nu}^{L Q}\right)}
$$

Here $N_{\mu \mu}$ and $N_{\mu \nu}$ are the upper limits on the number of expected events for

the respective two muon and single muon signatures. And $\epsilon_{\mu \mu}^{L Q}$ and $\epsilon_{\mu \nu}^{L Q}$ are the total respective detection efficiencies. The mass limit for the combined signature as a function of $\beta$ can be determined in a similar manner as the two muon signature mass limit.

The excluded branching ratio vs mass excluded region is shown in Fig. 4. The excluded region for the two muon signature is shown by the dotted line. The solid line shows the boundary of the excluded region for the combined signature. It can be seen that the single muon signature contribution to the detection efficiency significantly extends the mass vs branching ratio reach.

These results are in the process of being updated, review and verified, and it is intended that these results be written up for publication in the near future. A more detailed description of this analysis can be found in reference [5].

\section{References}

[1] F.E. Page and S. D. Protopopescu. Brookhaven National Laboratory Report No. BNL-37066, 1985, unpublished.

[2] R. Brun and F. Carminati. "GEANT Detector Description and Simulation Tod". Cern, Cern Program Library Long Writeup W5013, July, 1993. 


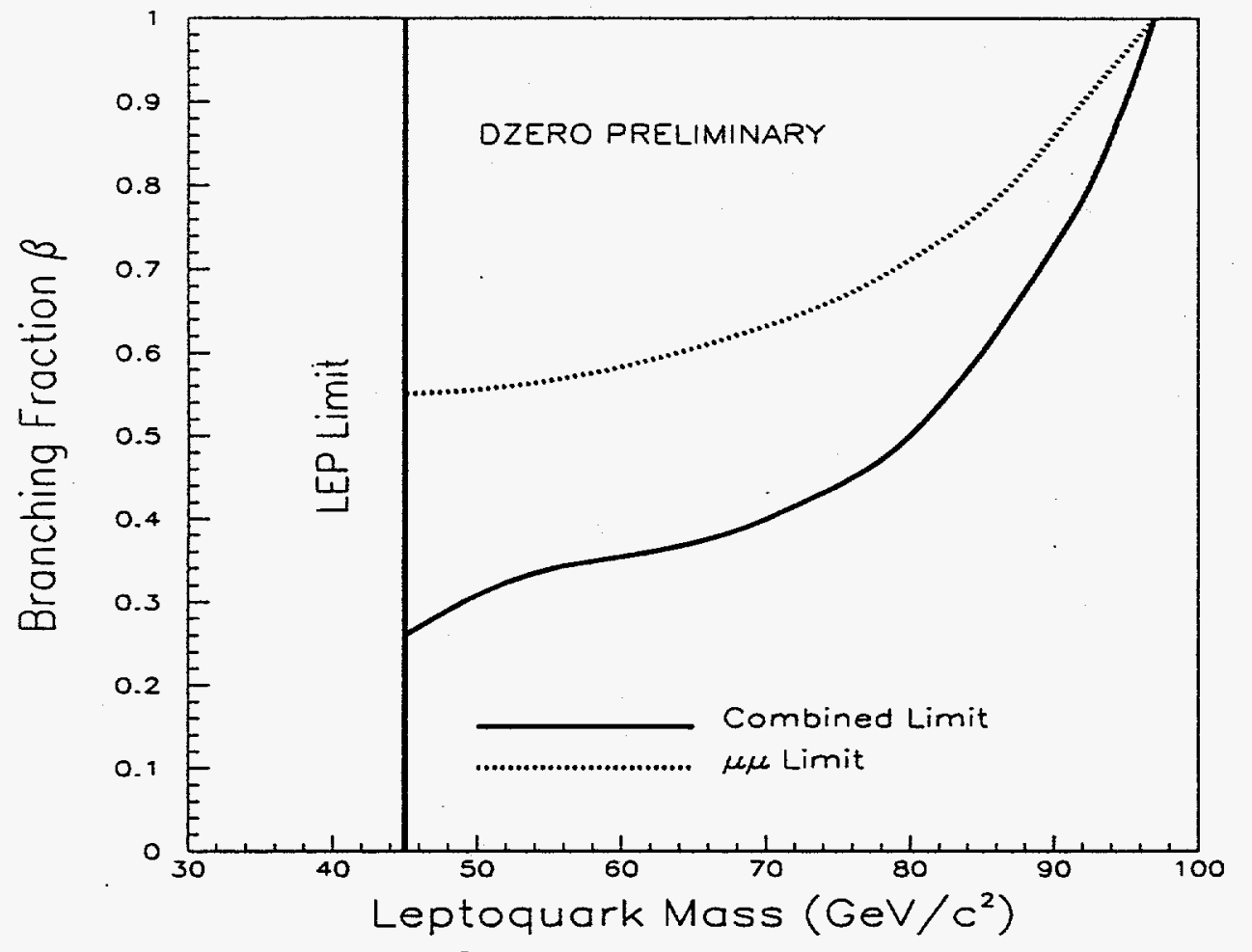

Figure 4: Ttre 95\% CL excluded region of branching fraction vs mass for second generätion scalar leptoquarks 
[3] R.D. Cousins and V.L. Highland. "Incorporating systematic uncertainties into the upper limit". Nucl. Instr. and Meth. A 320 (1992) pp.331335 .

[4] D. M. Norman. "A Search for First Generation Scalar Leptoquarks at $\sqrt{s}=1.8 \mathrm{TeV}$ with the $\mathrm{D} \emptyset$ Detector." Dissertation, The University of Maryland, 1993. D0note 1914. And references therein.

[5] D. M. Norman. "A Search for a Second Generation Leptoquark." DØ Note 2290, unpublished, Sept. 12, 1994.

\subsubsection{Other Physics}

Although the primary recent analysis work within our group has been concentrated on the Wino-Zino and leptoquark searches, major contributions to other analyses were also made. In this section we summarize these largest of these contributions.

\section{Large Et Events}

Jay Wightman, while a member of our group, began work with Henryk Piekarz in a study of extremely high Et events. The analysis is sensitive to a wide rąnge of new physics contributions including such sources as compositeness, new forces (Centauri events) and new heavy particles. This very interesting work was recently presented externally for the first time by Henryk Piekärz àt DPF '94.

Squärks and Gluinos $\rightarrow$ missing $\mathrm{Pt}+$ jets

One of the most important physics signatures at a hadron collider is missing transverse momentum. The best known reactions where this is involved 
include top, W and SUSY physics. In all cases, either a high Pt neutrino or the lightest SUSY particle is involved, so a large apparent momentum imbalance occurs. One member of our group, Amber Boehnlein (now a Fermilab employee) has contributed in the standard "missing Pt + jets" SUSY search. Her work was mainly concerned with the missing $\mathrm{Pt}$ trigger efficiency for the analysis. The full analysis was recently completed by Mark Paterno as his $\mathrm{PhD}$ thesis. The work has appeared in numerous conferences and is nearing publication.

$\mathrm{W} \rightarrow \tau \nu$

Amber also played a major role in the first efforts to measure the cross section of $W \rightarrow \tau \nu$ in $D \emptyset$. This reaction is extremely important for a number of reasons. In addition to a check of lepton universality, the ability to identify tau decays in $D \emptyset$ is important for potential Higgs searches and because tau's are an important source of background for new particle searches involving leptons. Here, her main contribution again involved the missing $\mathrm{Pt}$ signature, but she also was key contributor in the tau identification studies. (Student Djoko Wirjawan is now continuing this project by applying new techniques involving an H-Matrix.)

\subsection{Hardware and Software Responsibilities}

\subsubsection{Wino/Zino Triggers for Run 1B}

$$
=
$$

Much effort has gone into making sure that the $D \emptyset$ trigger used for the Wino/Zino searches are efficient for the signatures we look for. We have determined that some of the standard off-line electron quality cuts used to 
define good electrons were much more inefficient for low $\mathrm{E}_{t}$ electrons than for high $\mathrm{E}_{t}$ electrons. There was concern that the efficiency for analogous shape cuts used in the on-line software filters with low $E_{t}$ electron thresholds might suffer in a similar manner. The triggers used in the wino/zino search have the lowest thresholds of any unprescaled trigger, down to $7 \mathrm{GeV}$. We investigated the effect of the filter shape cuts and tried to determine if it were possible to remove or loosen the cuts if necessary and not effect the trigger rate too much. We found that it was possible to relax the cuts and not incur too much additional rate; however, it was also determined that it was unnecessary to do so. This was determined by processing the Monte Carlo electrons with added noise (to simulate the $D \emptyset$ detector environment) through a simulation of the $D \emptyset$ trigger. Monte Carlo electrons were used because there were no low $\mathrm{E}_{t}$ electron samples from collider data reasonably free from backgrounds to use in this study. It was determined that the effect of the on-line shape cuts on low $\mathrm{E}_{t}$ electrons was not as severe as the off-line shape cuts. The effect of the on-line isolation cut on a $45 \mathrm{GeV}$ wino - zino sample, taking into account the off-line kinematic cuts, was only about $5 \%$.

\subsubsection{Level-1 and Level-2 Trigger}

For the past. several years, our group has been deeply involved in the D $\emptyset$ trigger system. Two years ago, James White was a member of the trigger panel, and siñee then, Amber Boehnlein has been the New Phenomena group representative on the panel: She also serves as one of the two Trigger Meister. Panel members are responsible for developing the trigger lists and for 
determining rate quotas for the various terms. The Trigger Meister are responsible for maintaining the programmable triggering lists for the physics runs and for special runs. There are a large number of miscellaneous jobs and studies that are constantly being done to maintain and improve the trigger at the various stages. As an indication of the types of projects that have been done by our group, the following is a list of some of the highlights.

- Verification of Level 1 hardware: Compare trigger simulation with hardware trigger results.

- Verification of Level 2 software: Compare Level 2 results with fully reconstructed data (both real data and Monte Carlo data). Also compare results with previous versions.

- Timing studies of Level 2 software.

- Maintain rates files for trigger components to aid physics groups in developing new triggers.

- Study Level 1, Level'2 missing Pt efficiency including effect of Level 0 vertex position.

- Develop and maintain real time trigger monitoring software: While data is being collected, one of the shift tasks is to scan histograms from this monitoring program and compare with standards.

- Develop noise suppression software: developed software to recognize and remove "hot cells" from Level 2 triggering. 
This work was the primary responsibility of Amber Boehnlein during her tenure with our group. Students Djoko Wirjawan and Taylor Goss have joined this project at an entry level, and are now gaining the knowledge to contribute more strongly in the future.

An assortment of internal $D \emptyset$ documents ( $D \emptyset$ Notes) in this area include:

1. DØ Note 1220, "Level 1 Rates" Triggerfest, White/Boehnlein

2. $D \emptyset$ Note 1228, "Pt ..." - Triggerfest, White, J.

3. Dø Note 1235, "Supersymmetry.." -Triggerfest, White, A/White, J.

4. DØ Note 1334 "Level 1 and 2 rates for QCD backgrounds" Triggerfest 2, Boehnlein

5. DØ Note 1664, "Changes made to L2ETMISS and L2ETSUM during run Ia", Boehnlein

6. $D \emptyset$ Note 1724, "Trigger Studies for $W \rightarrow \tau \nu "$, Boehnlein

7. Dø Note 2174 , "A study of triggering on the missing Et at Level 1", Boehnlein

\subsubsection{Eevel-2 Software - Recent Student Contributions}

Over the past year, students Taylor Goss and Djoko Wirjawan have worked on several small projects while learning the system with guidance from senior personnel. A summary of these projects is now given. 
We have participated in a number of projects dealing with $D \emptyset$ 's Data Acquisition System, in particular the on-line software filter, which runs on a farm of parallel 4000 -series VAXstations. The filter is imposed after the hardware trigger, and is composed of a number of different tools which cut on various physics objects, e.g. an electron is required to pass a certain $E_{t}$ threshold and shape requirements, or an event is required to have a certain topology. New subroutines used in qualifying the physics objects continue to be written, and we check them prior to implementation in the filter.

All of the code development for the filter is done off-line, using a simulation of the filter. We also use the simulation to estimate the efficiency and rejection of the filter. There are many differences between the simulation and the actual filter, including the operating system under which each runs, and the way each manages memory. It is therefore important to verify that the simulation properly mimics the action of the on-line filter. A randomly selected sample of unbiased events which pass the hardware trigger are run through the simulation of the filter, and the results of the simulation are compared with the results of the actual filter by comparing the contents of the event data banks. We found and corrected several errors in the program which performs this comparison.

In addition to tasks directly related to the upkeep of the on-line filter; we have assisted others in settling filter issues relating to their specific physics interest. $\overline{\mathrm{I}} \mathrm{n}-\overline{\mathrm{S}} \mathrm{USY}$ Scalar Top decay, it was found that the jets tend not to be-collinear with each other or with the $\not_{T}$ in contrast to the leading backgrounds. We determined the optimal set of topological cuts to be used in the on-line filter for this search. In addition we independently verified the 
efficiency of proposed cuts for the Top to All Jets analysis.

We are actively involved in a SUSY particle search for the lightest chargino and the second lightest neutralino via the hadronically quiet tri-lepton decay channel. Since the leptons in this channel are typically low in $\mathrm{E}_{t}$ compared to electrons from $\mathrm{Z}^{0}$ 's, the preference would be to set the trigger thresholds well below those thresholds that are efficient for $\mathrm{Z}^{0}$ 's. However, the throughput of the filter is a limiting factor at high luminosities, which forces us to raise the thresholds to keep the rates from overwhelming the filter. This cuts into the efficiency of our search. We are currently engaged in a project to increase the throughput of the filter, so that the thresholds can be lowered and some of the lost efficiency regained.

We have contributed to the efforts of the on-line filter group in streamlining the on-line filter by writing code to optimize the usage of memory by the filter code and by verifying software used in compressing the electron filter data bank. This bank contains the various $E_{T}$ and quality parameters for each electron candidate as determined by the on-line filter electron algorithm. Off-line, we compare the electron filter bank built from its compressed form to the original bank to make sure that they both contain the same information before we drop the original bank. We have also contributed in the writing of utility subroutines for the printing of banks created by the hardware trigger. 


\subsubsection{Study of Electron Clustering Algorithms}

We worked with electron identification group to verify that using alternate clustering algorithms would result in a better $\mathrm{Z}$ mass peak resolution. We used $Z \rightarrow$ ee data from run $1 \mathrm{~A}$ with the uncorrected energy scale ( $Z$ mass centered at about $87 \mathrm{GeV}$ ) to see the effect of the alternate clusterings. Some of the clustering variables envisaged are listed in Table 3.2.4. The two alternate sets of variables, FIX and FIXET, are the choices that deviated the least from the current algorithm, $R E C O$, and showed promising improvement.

The variables used in Table 3.2.4 are defined as follows: Cluster_check if set to false will prevent summing two adjacent clusters, which share energy, into one cluster. Minimum_tower_energy is the minimum energy (in $\mathrm{GeV}$ ) of a tower to be added into a cluster. Cluster_energy_threshold is the minimum energy (in $\mathrm{GeV}$ ) for a cluster, while cluster_et_threshold is the minimum $E_{T}$ (in $\mathrm{GeV}$ ) for a cluster. Do_neighbor_energy if set to true will cluster towers in order of energy, otherwise will cluster towers in order of $E_{T}$. Cluster_etrans_threshold is the maximum fraction of the total cluster energy that is outside the cluster tower with the largest energy, while cluster_em_ratio_threshold is the minimum fraction of electromagnetic energy of a cluster.

There are 2108 events in the $Z \rightarrow$ ee data sample. Different clusterings as described in the Table 3.2.4 were applied to obtain three sets of data. We require the two highest $E_{T}$ electrons with $E_{T}>25 \mathrm{GeV}$ in each event to be of high quality. 
From $R E C O$ there are 363 events passing the $E_{T}$ and quality cuts, while from FIX and FIXET, respectively, there are 379 and 387 events. We calculated the invariant mass of the electron pairs and fitted the invariant mass distributions using a Breit-Wigner convoluted with a gaussian shape and approximated the background by a linear function. As an input for the Breit-Wigner fit, the $\mathrm{Z}$ width was set to $2.487 \mathrm{GeV}$ (the current value listed in the Particle Data Book), and the histograms were fitted around the Z mass peak window (from $70-110 \mathrm{GeV}$ ).

We do see some improvement of the $\mathrm{Z}$ mass peak resolution due to the alternate clusterings as shown in the following figures. Here is some conventions that are used in those figures. P1 is a normalization factor that represents the height of the peak, P2 and P3, respectively, are the mean value and the resolution of electron pairs' invariance mass. P4 and P5, respectively, are the y-intercept and the slope of the linear background. The upper plots in Fig. 5 - 3.2.4 are, respectively, the full range of invariant mass of the two highest electron pairs passing the required cuts, while the lower plots are their Breit-Wigner fits around the $\mathrm{Z}$ mass peak window.

\subsubsection{Global Monitoring Shift}

Amber has also been one of the organizers of the Global Monitoring shifts. This shiffistation was established to monitor the quality of the data as it is being collected. Her work included overseeing monitoring programs for triggering conditions, maintaining and upgrading a program for making histograms of triggered objects at all triggering levels, training the crew, and 

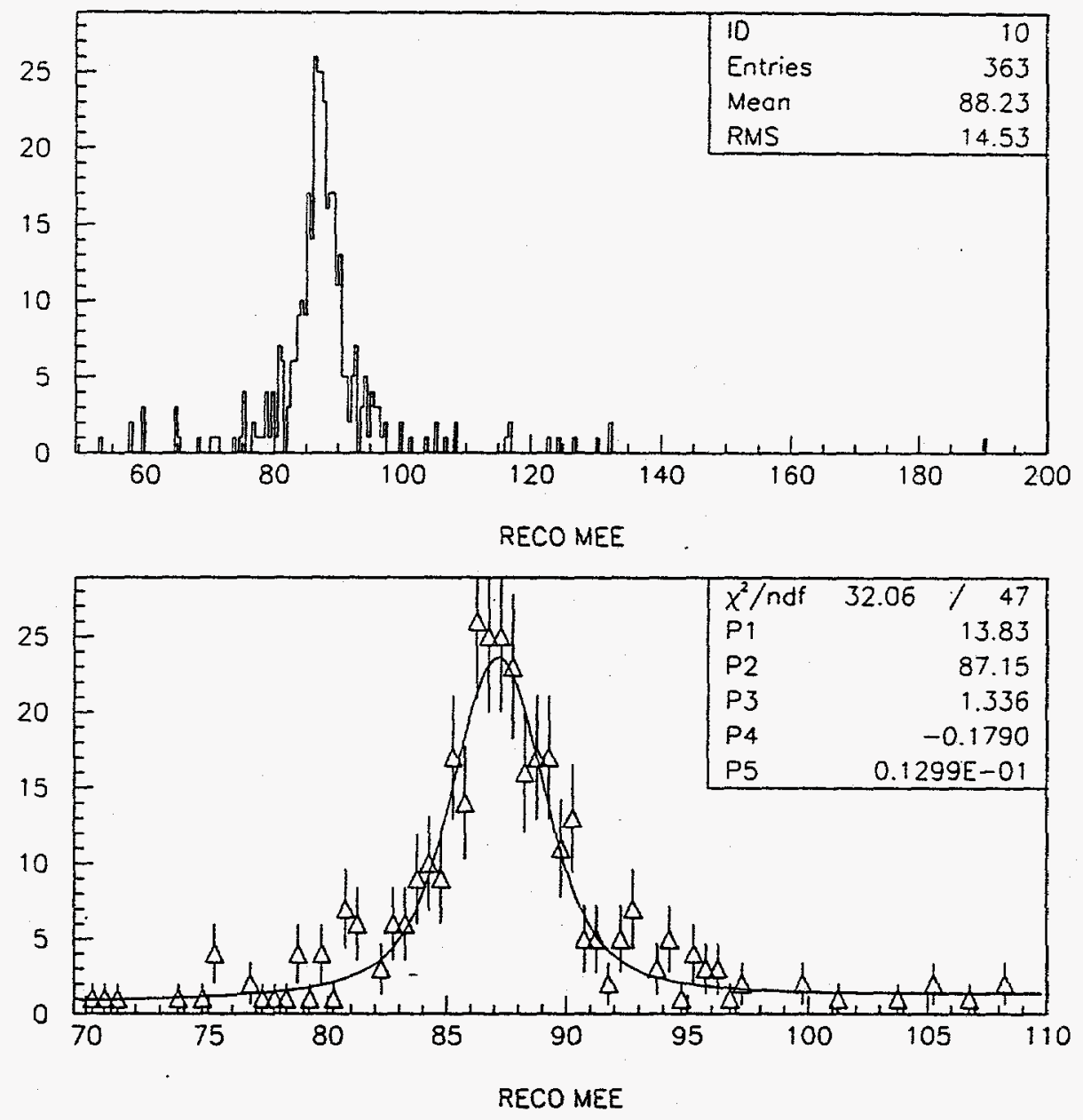

Figure 5: Invariance mass of electron pairs from $Z \rightarrow e e$ data using 'RECO' clustering-algorithm

$$
=-=
$$



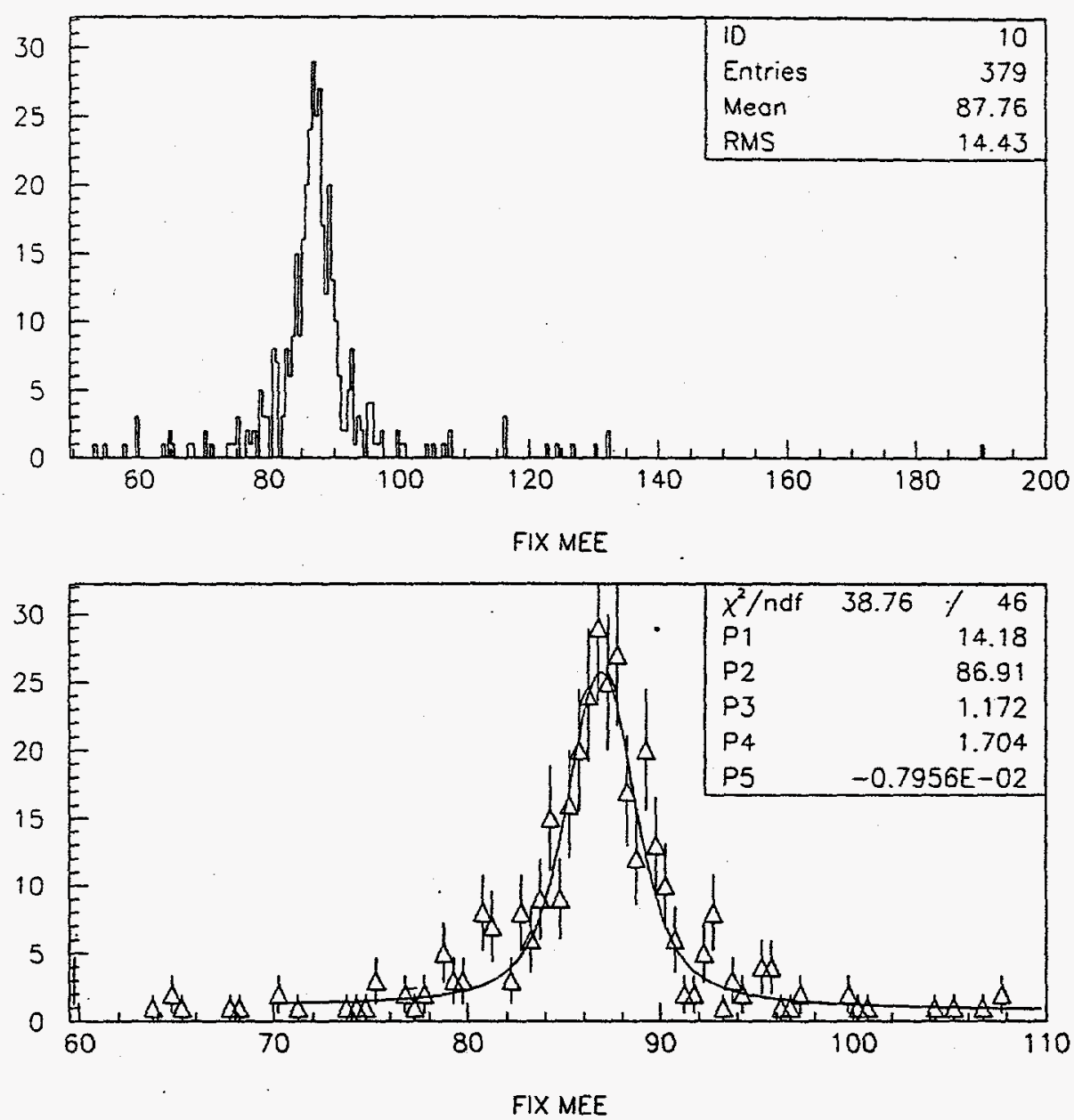

Figure 6: Invariance mass of electron pairs from $Z \rightarrow e e$ data using 'FIX' clustering algorithm

$=$ 


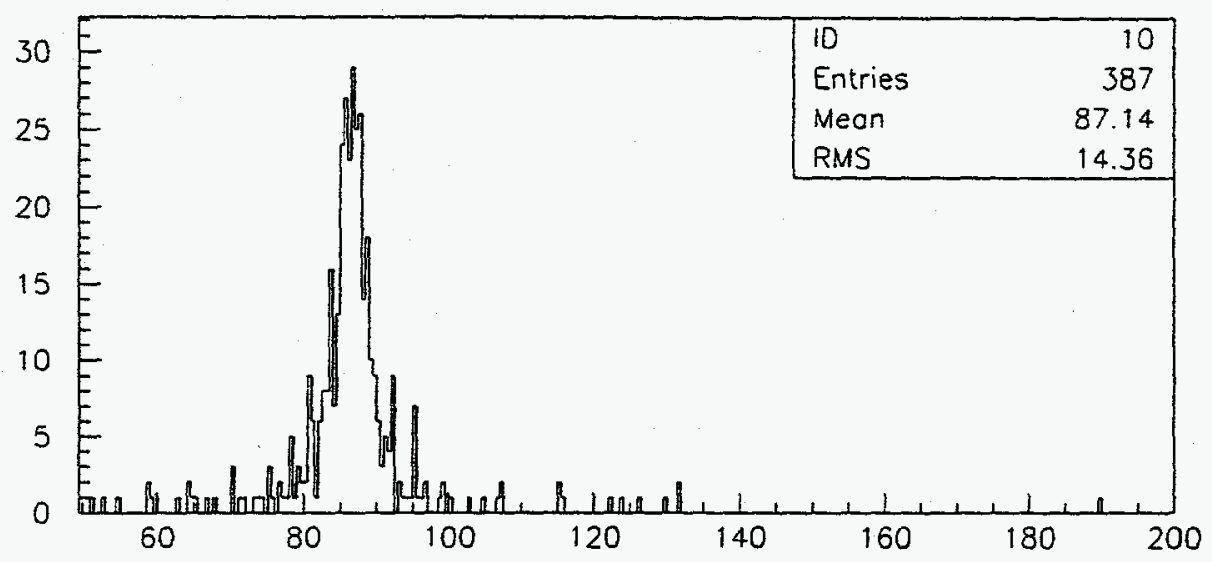

FIXET MEE

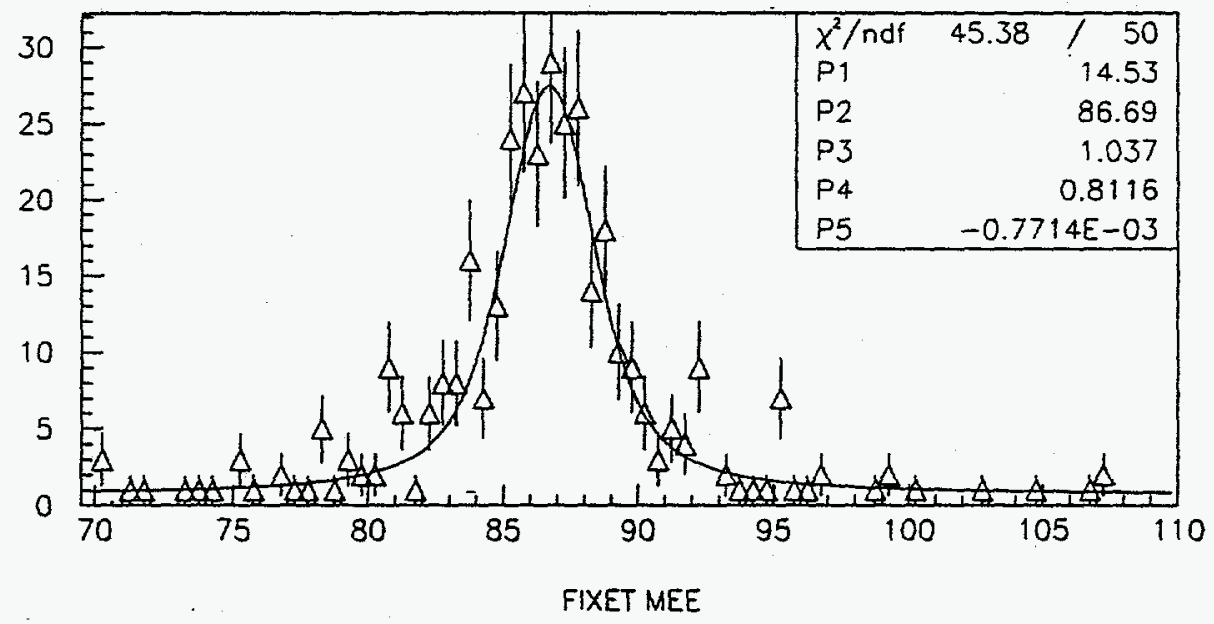

Figure 7: Invariance mass of electron pairs from $Z \rightarrow e e$ data using 'FIXET' clustering algorithm

$=$ 
providing additional information such as baseline histograms used by the crew to assess the quality of the data being collected.

Recent $D \emptyset$ Notes on this topic include:

1. DØ Note 1652, "Global Monitoring Training Notes", Bantly/Boehnlein/Klima

2. D $\emptyset$ Note 1652A, "Global Monitoring Documentation A Compilation", Bantly/Boehnlein/Klima,

\subsubsection{Data Processing}

Once the data is written, it must be reconstructed in a timely fashion. A group in $D \emptyset$ is responsible for doing this difficult job, and research associate Mei Gui undertook a six month tour of duty in this group from fall through spring 1994. The job included keeping track of tapes and processing them on the various computer farms at Fermilab. It also involves trouble shooting and personnel are on call around the clock.

\subsubsection{D $\emptyset$ Upgrade -}

In the early stages of the design of the upgrade detector, our group considered têchniques for fast triggering using the proposed fiber tracker. As an exereise, AND-OR circuit with all possible combinations from the fiber tracker for track Pt's greater than some specified limit. A hit pattern matching that of a high $\mathrm{Pt}$ track yielded an output so that the track could be matched to a calorimeter cluster or perhaps muon track. A chip was successfully designed 
and passed simulation tests, so that student $K$. Kotha was given a Master's degree in Electrical Engineering: Track Detection: an MCM ApproachMaster's Thesis, K. Kotha- 1993.

We are now looking at options concerning the forward region of the detector. These include tracking and possibly flling the intercryostat gap with an electromagnetic calorimeter.

\subsubsection{DØ Data Acquisition System Efforts}

The first project we undertook upon joining the $D \emptyset$ collaboration was to develop a package of routines for testing the data acquisition (DAQ) system hardware. At the start of this project, the DAQ hardware was almost non-existent. Since then, we have seen the DAQ system, both hardware and software, undergo major installation and upgrade to its present, hopefully stable, configuration. Throughout these changing times, our program has been maintaining pace with the installation and upgrades. We currently have a stand-alone program that is menu-driven and very user-friendly. It incorporates 3 major test packages which we call DOWNLOAD_TEST, IN_SITU_TEST, and VBD_TEST.

This work was the primary responsibility of Jay Wightman (now at Iowa State). The programs have the capability to test the Flash ADC's of the Central Tracker or the ADC's of the Calorimeter by loading test patterns in accessible buffers. The DAQ system can then be triggered so that the patterns coming out can be,compared with what went in. It is also possible to load full Monte Carlo events into the system and then trigger at high rates 
to study flow patterns. It is also to test individual boards including the crate controllers separately.

Details of this work have been presented in previous reports and elsewhere. A selection of major presentations and reports involving this work includes:

- "Computer Modeling of the D-Zero Data Acquisition System," R. Angstadt, M. Johnson, I. Manning, and J. Wightman, 1991 Nuclear Science Symposium and Medical Imaging Conference, Santa Fe, NM, November 2-9, 1992.

- "Modeling of the D-Zero Data Acquisition System," R. Angstadt, M. Johnson, I.L. Manning, and J.A. Wightman, IEEE Transactions on Nuclear Science $\underline{39}, 904$ (1992).

- "The D-Zero Data Acquisition System: Model and Measurements," J.A. Wightman, R. Angstadt, M.E. Johnson, and I.L. Manning, Workshop on DAQ/Trigger System Simulations for High Energy Physics, Dallas, TX, April 23-25, 1992.

- "Simulating the D-Zero Intermediate Hardware Trigger," M.E. Johnson, R. Angstadt, I.L. Manning, and J.A. Wightman, Workshop on DAQ/ Trigger System Simulations for High Energy Physics, Dallas, TX, April 23-25, 1992.

- "The D-Zero Data Acquisition System," J.A. Wightman, for the DZero Collaboration, $5^{\text {th }}$ International School-Seminar on Computing 
and Automation in Nuclear Physics and Astrophysics, Sochi, Russia, October 16-23, 1992. 


\begin{tabular}{|c|c|c|c|c|}
\hline Process & eee & $е е \mu$ & $e \mu \mu$ & $\mu \mu \mu$ \\
\hline Trigger & $\begin{array}{l}1 \mathrm{EM}>20 \\
\text { or } \\
2 \mathrm{EM}>10\end{array}$ & $\begin{array}{l}\mathrm{EM}>7, \mu>5 \\
\text { or } \\
2 \mathrm{EM}>20 \\
\text { or } \\
\mathrm{EM}>20, \not_{T}>20\end{array}$ & $\begin{array}{l}\mathrm{EM}>7, \mu>5 \\
\text { or } \\
\mu>15, \mu>10 \\
\text { or } \\
\mathrm{EM}>20, H_{T}>20\end{array}$ & $\begin{array}{l}1 \mu>15 \\
\text { or } \\
2 \mu>3\end{array}$ \\
\hline $\begin{array}{l}\text { Offline } \\
\text { Selection }\end{array}$ & $\begin{array}{l}3 e^{\prime} \mathrm{s},>7 \\
+\not_{T}^{\prime}>10\end{array}$ & $\begin{array}{l}1 \mu>10 \\
+1 e>10 \\
+1 e>7\end{array}$ & $\begin{array}{l}1 \mu>10 \\
+1 e>10 \\
+1 \mu>5 \\
+m_{\mu \mu}>5\end{array}$ & $\begin{array}{l}\text { e } \mu \text { 's }>5 \\
+m_{\mu \mu}>5\end{array}$ \\
\hline $\int L d t$ & $(14.8 \pm 1.8) \mathrm{pb}^{-1}$ & $(15.2 \pm 1.8) \mathrm{pb}^{-1}$ & $(15.2 \pm 1.8) \mathrm{pb}^{-1}$ & $(5.0 \pm 0.6) \mathrm{pb}^{-1}$ \\
\hline Result & 0 events & 1 event & 0 events & 0 events \\
\hline $\begin{array}{l}\text { Est. Bkg: } \\
\text { (PRELIM) }\end{array}$ & $F \quad 1.1$ events & $<0.5$ events & $<0.5$ events & $<0.2$ events \\
\hline
\end{tabular}

Table 1: Required trigger, offline selection criteria, integrated luminosity, number of events passing selection and estimated backgrounds for the four tri-lepton combinations. The background estimate for each channel is preliminary. 


\begin{tabular}{|l|l|l|l|}
\hline \hline clustering variables & RECO & FIX & FIXET \\
\hline cluster_check & $\mathrm{T}$ & $\mathrm{F}$ & $\mathrm{F}$ \\
minimum_tower_energy & 0.05 & 0.5 & 0.5 \\
cluster_energy_threshold & 1.5 & 1.5 & 1.5 \\
cluster_et_threshold & 1.5 & 1.5 & 1.5 \\
do_neighbor_energy & $\mathrm{T}$ & $\mathrm{T}$ & $\mathrm{F}$ \\
cluster_etrans_threshold & 0.6 & 0.6 & 0.6 \\
cluster_em_ratio_threshold & 0.9 & 0.9 & 0.9 \\
\hline \hline
\end{tabular}

Table 2: Summary of the current (RECO) and alternate (FIX and FIXET) clustering variables used in electron algorithms 


\section{The SDC Experiment at SSC}

Over the past few years, we worked on developing a new calorimeter technology involving liquid scintillating fibers in a spaghetti geometry for the forward detectors at the SSC. With the termination of the SSC, this work is now coming to an end. This work was highly successful and the technology (originally proposed by our group) was still a major contender for the forward calorimeter at the time the SSC was terminated. Our group made some of the key advancements in the technology. A list of major accomplishments is as follows:

- Established the fact that radiation induced discoloration of the cladding (in our case pyrex or quartz tubing) has no observable effect on light propagation.

- Found glass-liquid scintillator combinations with long attenuation lengths (exceeding 4 meters). This was a key finding that resolution requirements could be met with this technology! Also developed a test stand for the liquid fiber studies.

- Conducted studies of radiation damage to the liquid including in situ tests at the TAMU nuclear reactor. Established that the liquid is robust at leastup to 10 Mrads and can easily be circulated.

$$
=
$$

- Constructed lead-pyrex electromagnetic prototypes and tested in beam at Fermilab. (Including developing a technique for casting precision 1 $\mathrm{mm}$ holes in $30 \mathrm{~cm} \mathrm{~Pb}$ modules.) This work resulted in in the Master's 
thesis of Greg Dudgeon: "Liquid Scintillator Sampling Calorimetry", 1994.

- Constructed a $50 \mathrm{~cm}$ cast $\mathrm{Pb}-\mathrm{Sn}$ prototype. Technique involved casting welding rods covered with teflon tubing into large $\mathrm{Pb}-\mathrm{Sn}$ blocks. The rods could then be pulled by hand leaving smooth, precision holes.

- Constructed (with R. Webb) a $1.65 \mathrm{~m} \mathrm{Cu}$ plate hadronic prototype. This device is ready for beam tests and is currently being tested using cosmic rays.

- Constructed a 2-dimensional test stand for light guide development. Developed an efficient, uniform fiber light guide for use in liquid fiber calorimeters.

- Conducted numerous Monte Carlo studies to optimize calorimeter design.

As an example of some of our most recent work, we designed an optical fiber light guide for the large hadronic prototype constructed in collaboration with Bob Webb's group. It consists of a bundle of clear optical fibers with precision spacing on one side to match the liquid fibers, and a 'pony tail' on the other to be viewed by a phototube. A 4 " hexagonal light mixer sits between phototube and the fibers. A plot of the light transmission as a function of $\overline{\text { of }}$ fiber (position) is shown in Fig. 8. The light transmission measurement was done on our 2-d test stand. A computer controlled light source was moved in fine steps across each of the fibers and the phototube 

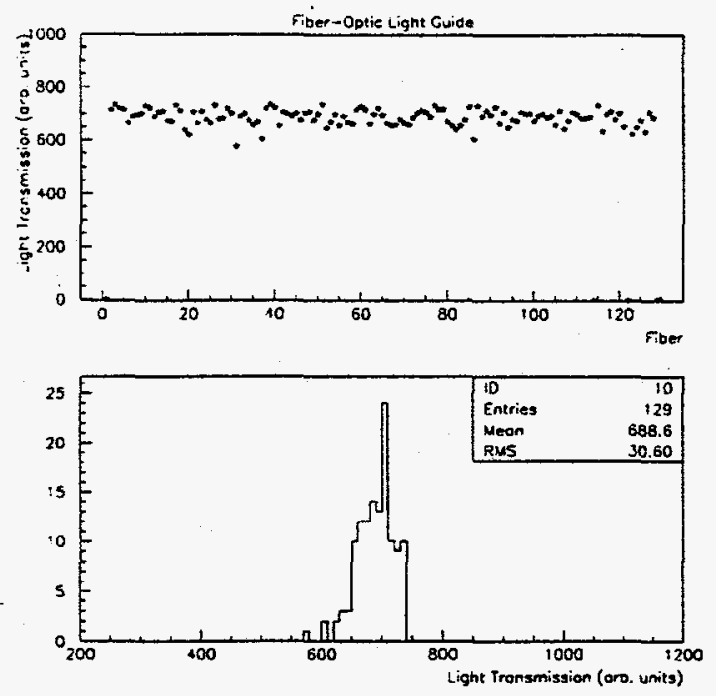

Figure 8: Measurement of light transmission through a prototype optical fiber light guide. Plotted is the light signal seen by a photomultiplier tube vs fiber number. A two dimensional computer controlled table was used to position a collimated LED at the center of each fiber. Except for damaged fibers, the signal varied less than $5 \%$ (sigma).

current was read out for each position. In this plot, the maximum light intensity for each fiber is plotted resulting in a $4.4 \%$ standard deviation in light response. This is much better than needed for SSC applications.

To summarize, our SSC detector work has been very successful, and the liquid fiber calorimeter is established as an excellent technology for extremely high radiation, high rate environments. A list of the presentations and publications at international conferences given over the past few years is given below. 
- 1) "Liquid SciFi Calorimeter", Workshop on Scintillating Fiber Detectors, October, 1993, Notre Dame, In.

- 2) "Liquid SciFi Calorimeter Prototype", Workshop on Scintillating Fiber Detectors, October, 1993, Notre Dame, In.

- 3) "Liquid Scintillating Calorimeter Prototype", Fall 1993 Joint Meeting of the Texas Section of the American Physical Society, College Station, TX.

- 4) "Measurement of Attenuation Length of Liquid Scintillating Fibers", Fall 1993 Joint Meeting of the Texas Section of the American Physical Society, College Station, TX.

- 5) "Measurement of Uniformity of Light Transmission in Light Guides for Liquid Scintillating Fiber Calorimeters", Fall 1993 Joint Meeting of the Texas Section of the American Physical Society, College Station, TX.

- 6) M. Gui, F. R. Huson, R. Pope and J. T. White. "Liquid Scintillating Fiber Calorimetry", Proceedings of SPIE's 1993 International Symposium on Optics, Imaging, and Instrumentation, July 1993, San Diego, $\mathrm{CA}$

- 7) Gui, F. R. Huson, T. Ostrovsky, D. Reese, D. Suson, and J.T. White, "Radiation Damage Effects on Liquid Scintillating Fibers", Radiat. Phys. Chem. Vol. 41, No. 1/2, pp. 237-242, 1993. 
- 8) M. Gui, F. R. Huson and J. T. White, "A Study of Glass-Liquid Scintillating Fibers for the Forward Calorimetry of the SDC", Proceedings of the 7th Meeting of the American Physics Society Division of Particles and Fields (DPF92), Nov. 1992, Fermi Lab, IL.

- 9) M. Gui, F. R. Huson, Z. Li, T. Ostrovsky, R. Pope, J.T. White, D. Suson, "Radiation Damage Study on Glass-liquid Scintillating Fibers", Proceedings of III International Conference on Calorimetry in High Energy Physics, Sept. 1992, Corpus Christi, TX.

- 10) P. Datte, et al., "A Liquid Scintillator Spaghetti Calorimeter EM Module for Forward Calorimetry at the SSC", Proceedings, Intnl Conf. on Calorimetry in High Energy Physics, Fermilab, Nov 1990 and Symposium on Detector Development for the Super Conducting Super Collider, Dallas, Oct 1990.

- 11) P. Datte, et al., "Effect of Radiation Induced Discoloration of the Cladding on Liquid Scintillating Fibers", Proceedings, Intnl Conf. on Calorimetry in High Energy Physics, Fermilab, Nov 1990 and Symposium on Detector Development for the Super Conducting Super Collider,-Dallas, Oct 1990.

We are also just now finalizing three papers that summarize these results for submisston to Nuclear Instruments and Methods. The titles of these papers are:

"Liquid Scintillating Fibers for Calorimetry", M. Gui, F.R.Huson, J.T. White (TAMU), D. Suson (TAM-Kingsville) 
"Radiation Damage Study on Glass-Liquid Scintillating Fibers", M. Gui, F.R.Huson, R. Pope, J.T White (TAMU), D. Suson (TAM-Kingsville) "Liquid Scintillating Fiber Calorimetry Prototype" M. Gui, D. Brookes, L. T. Goss, F.R. Huson, Y. Lu, R.C. Webb, and J.T. White

\section{Future}

Finally, our group has been very active in considering upgrade options for the future of Fermilab. In particular, we investigated the physics reach within the various upgrade options (high luminosity, increased energy or both) for topics relating to the search for supersymmetry. We found that one can reach up to $80 \%$ of possible SUSY phase space for both squarks-gluinos and charginos-neutralinos if one could double the Tevatron energy. Or, the reach would be similar for charginos-neutralinos with just a luminosity upgrade. This work is reported in the following documents:

1. "A Vision for High Energy Physics," T. Kamon, J.L. Lopez, P. Mcintyre and J.T. White, CTP-TAMU-11/94 1994 (unpublished).

2. "Supersymmetry at a proposed $\sqrt{s}=4 \mathrm{TeV}$ upgrade of the Fermilab Tevation". T. Kamon, J.L. Lopez, P. Mcintyre and J.T. White, Phys. Rex 0 ए 50 (1994) 5676

$$
=-
$$

3. "Tevatron Energy and Luminosity Upgrades Beyond the Main injector", D. Amidei it et al., presented at the Eight Meeting of the Division of Particles and Fields, New Mexico, 1994. Fermilab-Conf-94/249 


\section{Summary}

Since joining $D \emptyset$ in 1990 , our group has participated as strongly as possible in all phases of the experiment. The commissioning of the electronics and trigger system, and development of an efficient triggering scheme was central to the effort that lead to the success of $D \emptyset$ 's first data run. Our group played a major role in these efforts. We have also been highly active in initiating and extracting exciting physics from the data. Our two $\mathrm{PhD}$ students are now trained in the "DØ software experience" and are begining to make major contributions. We feel our work has met obligations, and we are looking forward to continuing this level of activity in the future. 